\title{
Woolly hair generalizado: caso clínico e revisão da literatura
}

\author{
Generalized woolly hair: case report and literature review
}

\author{
Tiago Torres ${ }^{1}$ \\ Manuela Selores ${ }^{3}$
}

Susana Machado ${ }^{2}$

\begin{abstract}
Resumo: O termo woolly hair, ou cabelo lanoso, refere-se a uma variante anormal de cabelo, que pode ser parcial ou generalizado. As variantes de woolly hair generalizado são raras e podem ocorrer isoladamente ou associadas a outras alterações cutâneas e extracutâneas. Desta forma, nestes doentes, é necessário a exclusão de certos síndromes, como o de Noonan e cardiofaciocutâneo (CFC), que cursam com importante morbilidade e mortalidade. É descrito o caso clínico de uma criança com woolly hair generalizado e queratose pilar, mas que, após avaliação dos vários órgãos e sistemas, não foi encontrada qualquer alteração ou característica associada a esses síndromes.

Palavras-chave: Ceratodermia palmar e plantar; Doenças do cabelo; Síndrome de Noonan

Abstract: The term woolly hair, or tightly curled hair, refers to a structural anomaly of scalp hair, which can be partial or generalized. Generalized woolly hair variants are rare and can occur in isolation or in association with other cutaneous and extra-cutaneous abnormalities. Thus, it is important to dismiss in these patients the diagnosis of syndromes such as the Noonan syndrome and Cardiofaciocutaneous syndrome, which have high morbidity and mortality rates. We report the clinical case of a 7 year-old boy with generalized woolly hair and keratosis pilaris. After an evaluation of organs and systems, no alteration or characteristics associated with these syndromes were found.

Keywords: Palmoplantar keratoderma; Hair diseases; Noonan syndrome
\end{abstract}

\section{INTRODUÇÃO}

O termo woolly hair refere-se a uma variante anormal de cabelo, fino, enovelado e geralmente hipopigmentado. Foi inicialmente descrito por Gossage $^{1}$ em 1907 e posteriormente, em 1974, Hutchinson $e t ~ a l .^{2}$ classificaram o woolly hair em três variantes: uma variante localizada, designada de woolly hair nevus, e duas variantes generalizadas, denominadas de woolly hair hereditário autossómico dominante e woolly hair familiar autossómico recessivo. As variantes de woolly hair generalizado podem ocorrer isoladamente ou fazer parte de doenças genéticas, como o síndrome de Noonan, o síndrome cardiofaciocutâneo (CFC) ou a doença Naxos, em associação a anomalias cutâneas, como queratose pilar ou queratodermia palmoplantar, e extracutâneas, sobretudo cardíacas ou dos órgãos dos sentidos.

\section{RELATO DO CASO}

Uma criança do sexo masculino, de 7 anos de idade, caucasiano, recorreu à consulta de dermatologia por apresentar desde o primeiro ano de vida hipotricose generalizada. Referia, igualmente, uma diminuta taxa de crescimento do cabelo, que impossibilitava o crescimento do cabelo superior a alguns centímetros. Ao exame objectivo observava-se uma criança saudável, com um desenvolvimento psicomotor normal, uma morfologia facial normal (nomeadamente normal implantação das orelhas, dentição normal, normotelorismo), e, envolvendo todo o couro cabeludo, cabelo castanho fino, macio, enovelado e curto, que permitia a observação do couro cabeludo, este sem alterações (Figura 1). A nível da face externa dos membros e nuca observavase marcada queratose pilar. $O$ restante exame dermatológico era normal.

\footnotetext{
Recebido em 03.03.2009.

Aprovado pelo Conselho Consultivo e aceito para publicação em 16.06.09.

* Trabalho realizado no Serviço de Dermatologia do Centro Hospitalar do Porto - Hospital de Santo António, EPE - Porto, Portugal.

Conflito de interesse: Nenhum / Conflict of interest: None

Suporte financeiro: Nenhum / Financial funding: None

Interno de Dermatovenereologia; Serviço de Dermatologia do Centro Hospitalar do Porto, Hospital de Santo António - Porto, Portugal.

Especialista de Dermatovenereologia; Serviço de Dermatologia do Centro Hospitalar do Porto, Hospital de Santo António - Porto, Portugal.

Professora regente da disciplina de Dermatologia na Faculdade de Biomédicas da Universidade do Porto; directora de Serviço; Serviço de Dermatologia do

Centro Hospitalar do Porto - Hospital de Santo António - Porto, Portugal.
} 


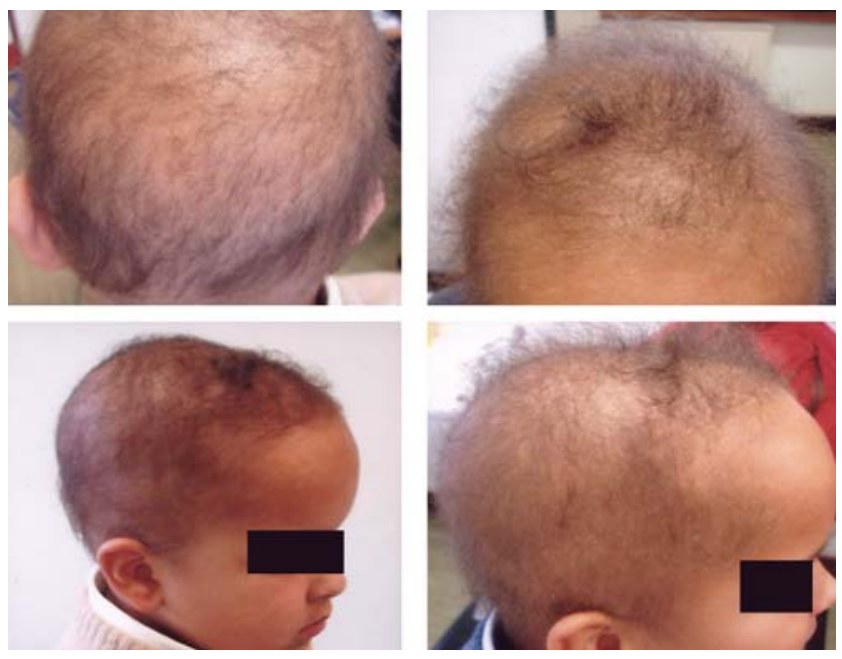

Figura 1: Cabelo castanho fino, enovelado e curto envolvendo todo o couro cabeludo
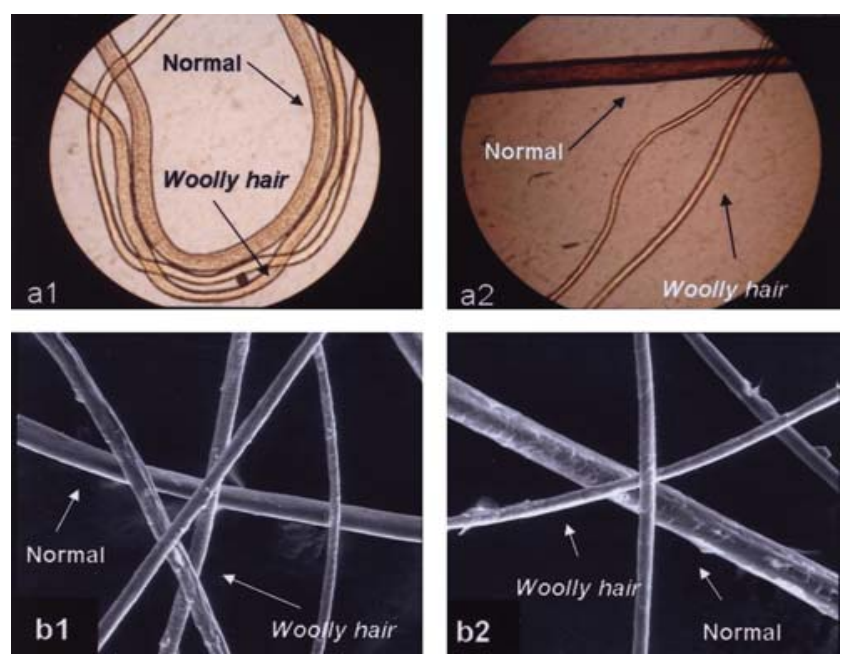

FigurA 2: Comparação, à microscopia óptica (a1 e a2) e electrónica (b1 e b2), de woolly hair do doente e de cabelo normal

Wise et $a .^{3}$, na grande maioria dos doentes, esta displasia pilosa inicia-se nos primeiros anos de vida e, em cerca de 50\% dos casos, associa-se a nevo epidérmico linear ou nevo verrucoso, que pode, ou não, envolver a área do cabelo afectadó. Pode ocorrer envolvimento ocular, incluindo membrana pupilar persistente ou perda localizada de pigmento retiniano, ${ }^{5}$ pelo que o exame oftalmológico está sempre indicado nestes doentes.

O síndrome woolly hair nevus caracteriza-se pela presença desta anomalia capilar associada a nevo epidérmico e outras anomalias extracutâneas, destacando-se alterações ósseas, neurológicas, oftalmológicas e, menos frequentemente, cardíacas e renais. ${ }^{6} \mathrm{O}$ woolly hair generalizado envolve todo o couro cabeludo, podendo ocorrer isoladamente ou associado a outras alterações cutâneas e/ou extracutâneas.

A presença de dismorfia facial caracteriza muitas das doenças associadas a woolly hair. Doentes com síndrome de Noonan exibem uma face característica que inclui implantação baixa das orelhas, pregas epicânticas e hipertelorismo. Outras características importantes envolvem baixa estatura, surdez e anormalidades cardíacas (especialmente estenose pulmonar). ${ }^{7} \mathrm{O}$ síndrome $\mathrm{CFC}$ caracteriza-se por uma face semelhante à encontrada no síndrome de Noonan: atraso de crescimento, ictiose, coiloníquia e defeitos estruturais cardíacos. ${ }^{8}$

A queratose pilar faz parte de um grupo de genodermatoses que inclui uleritema ofriogenes $\mathrm{e}$ atrofoderma vermiculatum e, tal como o woolly hair, também é encontrada nos síndromes $\mathrm{CFC}^{9,10}$ e de Noonan. No entanto, está descrita a presença de woolly hair generalizado e queratose pilar na 
ausência de síndrome $\mathrm{CFC}^{9}$ ou de Noonan.

A tríade woolly hair generalizado, queratodermia palmoplantar e cardiomiopatia ventricular direita define a doença de Naxos,${ }^{11}$ uma doença autossómica recessiva rara, associada, na maioria dos casos, à mutação de uma proteína desmossómica, denominada de placoglobina. ${ }^{12} \mathrm{O}$ woolly hair aparece logo após o nascimento, a queratodemia palmoplantar desenvolve-se no primeiro ano de vida e a cardiomiopatia manifesta-se clinicamente na adolescência, com síncope, taquicardia ventricular, falência cardíaca direita ou morte súbita.

Mutações de outra proteína desmossómica, a desmoplaquina, ${ }^{13}$ estão associadas ao síndrome de Carvajal, ${ }^{14}$ caracterizado por woolly hair generalizado, queratodermia palmoplantar e cardiomiopatia dilatada. A doença cardíaca manifesta-se ainda na infância e envolve, geralmente, o ventrículo esquerdo. Desta forma, na presença de woolly hair generalizado, deve ser feita uma avaliação completa de todos os órgãos e sistemas, particularmente cardíaco.

No woolly hair, o diâmetro capilar é menor do que o do cabelo normal e, em secção transversal, o cabelo é oval, não redondo. A taxa de crescimento é normal, mas, geralmente, incapaz de promover um crescimento superior a alguns centímetros. $\mathrm{O}$ rácio anagénese-telogénese é normal, mas o período de anagénese é menor, além da raiz na fase anagénica ser geralmente distrófica e sem bainha. A fragilidade do cabelo não está aumentada, excepto quando associado a tricorrexis nodosa (comum na variante hereditária dominante e muito rara na variante familiar recessiva). ${ }^{2}$ A presença de pili annulati é mais comum, observando-se à microscopia de luz bandas escuras e claras alternadas. A variante localizada, woolly hair nevus, pode apresentar outras alterações, especialmente, perda da cutícula, tricorrexis nodosa e vacuolização cortical. Não existe qualquer tratamento eficaz, mas, em alguns doentes, o cabelo pode tornar-se mais escuro e menos enovelado com a idade.

Nesta criança, a presença de woolly hair e de queratose pilar levou a considerar o diagnóstico de síndrome de Noonan ou CFC e à exclusão das principais comorbilidades associadas a estes síndromes. A ausência de outras alterações afasta estes diagnósticos e sugere que se trate de uma outra genodermatose, semelhante à descrita, em 1984, por Neil et al. numa família com woolly hair e uleritema ofriogenes, na ausência de síndrome de Noonan. ${ }^{9} \mathrm{O}$ presente caso clínico alerta para a importância da associação de alterações do cabelo com anomalias de outros órgãos e a necessidade da sua exclusão. 


\section{REFERÊNCIAS}

1. Gossage AM. The inheritance of certain human abnormalities. Q J Med. 1907;1:331-47.

2. Hutchinson PE, Cairns RJ, Wells RS. Woolly hair: clinical and general aspects. Trans St Johns Hosp Dermatol Soc. 1974;60:160-77.

3. Wise F. Woolly hair nevus. A peculiar form of birthmark of hair of the scalp, hitherto undescribed, with report of two cases. Med J Rec. 1927;125:545-7.

4. Bovenmeyer DA. Woolly hair nevus. Cutis. 1979;24:322-5.

5. Jacabsen KU, Lowes M. Woolly hair nevus with ocular involvement: a report of a case. Dermatologica. 1979;151:249-52.

6. Al Harmozi SA, Mahmoud SF, Ejeckam GC. Woolly hair nevus syndrome. J Am Acad Dermatol. 1992;27:259-60.

7. Burton JL. The genes for Noonan's syndrome, woolly hair and ulerythema ophryogenes. Postgrad Med J. 1992;68:595-9.

8. Manoukian S, Lalatta F, Selicorni A, Tadini G, Cavalli R, Neri G. Cardio-facio-cutaneous syndrome: report of an adult without mental retardation. Am J Med Genet. 1996;63:382-5.

9. Neild VS, Pegum JS, Wells RS. The association of keratosis pilaris atrophicans and woolly hair, with and without Noonan's syndrome. $\mathrm{Br} \mathrm{J}$ Dermatol. 1984;110:357-62.

10. McHenry PM, Nevin NC, Bingham MB. The association of keratosis pilaris atrophicans with hereditary woolly hair. Pediatr Dermatol. 1990;7:202-4.

11. Rai R, Ramachandran V, Sundaram G, Rajendren C, Srinivas R. Naxos disease: a rare occurrence of cardiomyopathy with woolly hair and palmoplantar keratoderma. Indian J Dermatol Venereol Leprol. 2008;74:50-2.
12. McKoy G, Protonotarios N, Crosby A, Tsatsopoulou A, Anastasakis A, Coonar A, et al. Identification of a deletion in plakoglobin in arrhythmogenic right ventricular cardiomyopathy with palmoplantar keratoderma and woolly hair (Naxos disease). Lancet. 2000;17:2119-24.

13. Norgett EE, Hatsell SJ, Carvajal-Huerta L, Cabezas JC, Common J, Purkis PE, et al. Recessive mutation in desmoplakin disrupts desmoplakin-intermediate fila ment interactions and causes dilated cardiomyopathy, woolly hair and keratoderma. Hum Mol Genet. 2000;9:2761-6.

14. Carvajal-Huerta L. Epidermolytic palmoplantar keratoderma with woolly hair and dilated cardiomy opathy. J Am Acad Dermatol. 1998;39:418-21.

\footnotetext{
ENDEREÇO PARA CORRESPONDÊNCIA / MAILING ADDRESS:

Tiago da Costa Ferreira Torres Serviço de Dermatologia do Centro Hospitalar do Porto - Hospital de Santo António, EPE

Edifício das consultas externas, ex-Cicap,

Rua D. Manuel II. 4100 Porto, Portugal

Tel./Fax: 226097429226097429

E-mail: tiagotorres2002@hotmail.com
} 